Reprod. Nutr. Dévelop., 1983, 23 (5), 907-914.

\title{
Etude de la gastrine chez Gallus gallus. I. - Mise en évidence d'une immunoréactivité et d'une activité biologique de type gastrine dans le tractus digestif de poulets
}

Nicole RIDEAU, P. MONGIN

Station de Recherches avicoles, I.N.R.A. Nouzilly 37380 Monnaie, France.

Summary. A study of gastrin in Gallus gallus. I. Evidence of gastrin-like immunoreactivity and biological activity in chicken digestive tract.

Extracts of the digestive tract of the chicken were examined for gastrin-like material. An heterologous radioimmunological system using antihuman gastrin 1.17 antibodies permitted the quantitative determination of immunoreactive gastrin along the digestive tract. Immunoreactivity was restricted to the pyloric junction. This tissue contained $18.5 \pm 2.5$ $(n=6) \mathrm{ng}$ of equivalent human gastrin (1.17) per $\mathrm{g}$ wet weight. After gel filtration, immunoreactivity occurred in three molecular forms which cleaved to one heptadecapeptide gastrin-like form by tryptic cleavage. Gastrin bioactivity of the pyloric extract was measured in anesthetized chickens. Extracts containing $240 \mathrm{pg}$ of gastrin-like immunoreactivity had a biological activity corresponding to about $10 \mu \mathrm{g}$ of pentagastrin, suggesting that pentagastrin is weakly active in the chicken as well as in mammals and/or that avian gastrin was not fully recorgnized by the radioimmunological system used.

\section{Introduction.}

La régulation neuroendocrinienne de l'appareil digestif reste très mal connue chez les oiseaux. Plusieurs auteurs ont recherché l'effet des hormones mammaliennes (Kokas et al., 1967, 1971 ; Burhol, 1971 ; Sewing et Ruoff, 1972 ; Dockray, $1975 ;. .$.$) mais il y a un manque évident d'information concernant la caracté-$ risation, la signification physiologique, voire même l'existence de certaines hormones gastrointestinales aviaires (Hazelwood, 1980).

En particulier la présence de gastrine dans le tube digestif des oiseaux a longtemps été controversée (Blair et al., 1967 ; Ruoff et Sewing, 1970 ; Gabe, 1972 ; Olowo-Okorun et Amure, 1973 ; Ketterer et al., 1973 ; Polak et al., 1974 ; Andrew, 1976). Ce n'est qu'en 1974 que Larsson et al., I'ont définitivement localisée chez le poulet par microscopie électronique et par techniques immunohistologique et radioimmunologique, dans une zone étroite $(0,5-1 \mathrm{~cm})$ située à la jonction du gésier et du duodénum. Linari et Baldieri-Linari (1976) ont par la suite apporté une preuve indirecte de l'existence d'une substance " gastrine-like " en 
montrant que des poulets privés de jonction pylorique présentent une secrétion gastrique basale réduite et non stimulable par la Bombesine.

Dans cette note nous apportons des précisions concernant la caractérisation immunologique et chromatographique de la gastrine dans des extraits de jonction gastroduodénale de poulet (Gallus gallus) et nous examinons l'activité gastrosecrétoire de ces extraits chez le poulet.

\section{Matériel et méthodes.}

1. Préparation des extraits de muqueuse digestive. - On prélève immédiatement après l'abattage des poulets (de souche Warren, âgés de 5 à 8 semaines), différentes portions du tube digestif : jabot, proventricule, jonction gastro-duodénale, duodenum, jejunum. Ces fragments tissulaires sont rapidement lavés à l'eau froide, pesés et congelés à $-40^{\circ} \mathrm{C}$, puis traités selon la méthode de Blair et al. (1961) pour l'extraction de la gastrine des mammifères : les extraits aqueux sont soumis $30 \mathrm{~min}$ au bain marie bouillant, les surnageants sont ensuite concentrés par précipitation à l'acétone. Les échantillons sont stockés à $-20^{\circ} \mathrm{C}$.

2. Dosage radioimmunologique de la gastrine aviaire. - On utilise la trousse fournie par le Commissariat à I'Energie Atomique (CEA, Saclay, France) pour le dosage radioimmunologique de la gastrine : elle contient un antisérum lobtenu chez le lapin par injection de gastrine humaine synthétique 1.17 couplée à de l'albumine bovine) utilisé à la dilution finale $1 \times 10^{-6}$ et dè la gastrine humaine synthétique 1.17 utilisée comme référence et comme traceur radioactif $\left({ }^{125}\right)$. La séparation de la gastrine libre et liée est réalisée par adsorption de l'hormone libre sur charbon Norit A-dextran T 70 après $96 \mathrm{~h}$ d'incubation à $4{ }^{\circ} \mathrm{C}$. Tous les réactifs sont conservés à l'état lyophilisé et ils sont reconstitués au moment de l'emploi avec un tampon véronal 0,02 $\mathrm{pH} \mathrm{8,4}$.

La spécificité de l'anticorps antigastrine humaine 1.17 définie par le pourcentage de réaction croisée avec les substances d'origine humaine de structure proche est respectivement de $100,73,38,3,5$ et $3,5 \%$ pour les gastrine 1.17 , gastrine 2.17, big-gastrin, cholecystokinine et pentagastrine (référence trousse Gask CEA). Les critères de validité de ce dosage homologue appliqué à l'espèce aviaire sont présentés plus loin.

Afin de rechercher les formes de gastrine présentes dans la jonction pylorique, des aliquots d'extraits bruts $(1 \mathrm{ml})$ sont chromatographiés sur colonne de séphadex $\mathrm{G} 50$ superfine $(100 \times 1,1 \mathrm{~cm})$ avant et après digestion trypsique (trypsine à $1 \mathrm{mg} / \mathrm{ml}$ dans du tampon phosphate $0,25 \mathrm{M} \mathrm{pH} \mathrm{6,8,} \mathrm{incubation} \mathrm{de}$ $0,25 \mathrm{mg}$ de trypsine par $\mathrm{ml}$ d'extrait $10 \mathrm{mn}$ à $37^{\circ} \mathrm{C}$, la réaction est stoppée par chauffage à $100^{\circ} \mathrm{C}(10 \mathrm{mn})$ (Rehfeld, 1972). La colonne est équilibrée et éluée avec du tampon véronal $0,02 \mathrm{M} \mathrm{pH} \mathrm{8,4} \mathrm{contenant} \mathrm{de} \mathrm{l'azoture} \mathrm{de} \mathrm{sodium} \mathrm{à} 2$ pour mille ; elle est calibrée à $20^{\circ} \mathrm{C}$ à l'aide de bleu dextran, de gastrine humaine 1.17 iodée $\left({ }^{125}\right)$ et de chlorure de sodium ; le débit est de $15 \mathrm{ml} / \mathrm{h}$. Des fractions de $2 \mathrm{ml}$ sont recueillies puis stockées à $-20^{\circ} \mathrm{C}$ avant dosage radioimmunologique. 
3. Dosage biologique. - Le pouvoir secrétoire gastrique des extraits pyloriques est étudié selon la méthode de Goto et Watanabe (1976) sur des jeunes poussins (de souche JV 15) âgés de 12 à 14 jours et anesthésiés à l'aide d'uréthane $(1,2 \mathrm{~g} / \mathrm{kg} \mathrm{IP})$. Les estomacs sont perfusés toutes les $15 \mathrm{~min}$ avec $2 \mathrm{ml}$ $\mathrm{d}^{\prime}$ une solution de chlorure de sodium à $9 \mathrm{~g} / \mathrm{l}$ à $37^{\circ} \mathrm{C}$; les perfusats recueillis à la sortie du gésier sont titrés à $\mathrm{pH} 7,4$ à l'aide d'un titrateur automatique (Radiometer, Copenhague) avec de la soude $0,01 \mathrm{~N}$. Les résultats sont exprimés en $\mu \mathrm{Eq}$ $\mathrm{H}+/ 15 \mathrm{mn} / 50 \mathrm{~g}$ de poids vif. Les extraits aviaires et la pentagastrine utilisée comme substance de référence sont administrés par voie intraveineuse directe.

Les produits chimiques utilisés sont les suivants: trypsine (Calbiochem 64852) ; urethane (Sigma 4-2500) ; pentagastrine (Calbiochem 34763) ; sephadex G50 superfine (Pharmacia); bleu dextran 2000 (Pharmacia) ; trousse Gask pour le dosage radioimmunologique de la gastrine humaine ICEA-SORIN, Saclay, France).

\section{Résultats.}

1. Validité du dosage radioimmunologique appliqué à l'espèce aviaire et étude des formes de gastrine. - La sensibilité du dosage lou concentration seuil provoquant une inhibition significative $(P<0,01)$ de la liaison antigèneanticorps) est de $2,5 \mathrm{pg}$ équivalents gastrine humaine $1.17 \mathrm{par} \mathrm{ml}$; la variabilité intradosage est de $13 \%$ pour un même échantillon d'extrait pylorique dosé 10 fois, la variabilité interdosage varie de 17 à $20 \%$ pour des échantillons dosés lors de 7 manipulations différentes.

La validité du dosage d'extraits aviaires en système hétérologue a pu être évaluée par un test de parallélisme. Des dilutions croissantes d'extraits de jonction pylorique aviaire (fig. 1) se comportent vis-à-vis de l'anticorps antigastrine humaine 1.17 de la même manière que des dilutions du standard gastrine humaine 1.17 (test de parallélisme $P<0,05$ ). L'anticorps reconnaît donc une immunoréactivité de type gastrine dans la zone pylorique. Le tableau 1 montre que cette région est de loin la plus riche en gastrine immunoréactive ; les activités mesurées correspondent à une teneur moyenne de $18,5 \pm 2,5\left(\mathrm{~m} \pm \mathrm{s}_{\mathrm{m}}\right.$, $n=6) \mathrm{ng}$ équivalents gastrine humaine 1.17 par gramme de matière fraîche.

TABLEAU 1

Gastrine immunoréactivité présente dans différents extraits $\left(^{*}\right)$ de muqueuse digestive chez la poule (exprimée en ng équivalents de gastrine humaine 1.17 par $\mathrm{g}$ de matière sèche).

\begin{tabular}{ll}
\hline Jabot & 0,002 \\
Proventricule & 0,007 \\
Jonction gastroduodénale & 1,700 \\
Duodénum & 0,023 \\
Jéjunum & 0,008 \\
\hline
\end{tabular}

$\left(^{*}\right)$ Les extraits sont préparés (selon la méthode de Blair et al., 1961) à partir d'un pool d'organes prélevés sur 15 poules Warren. Chaque extrait a été titré à 2 dilutions différentes. 
Pour caractériser la substance immunoréactive, nous avons fractionné sur colonne de sephadex G50 superfine un extrait de jonction gastroduodénale aviaire avant et après digestion trypsique. La figure 2 montre les profils d'élution obtenus. Avant digestion trypsique, le matériel immunoréactif se répartit en 3 pics d'égale importance dont le plus tardif correspond à l'élution de la $\left.\right|^{125}$ gastrine humaine 1.17. Après action de la trypsine l'immunoréactivité est principalement éluée en même temps que la $\left.\right|^{125}$ gastrine humaine 1.17.
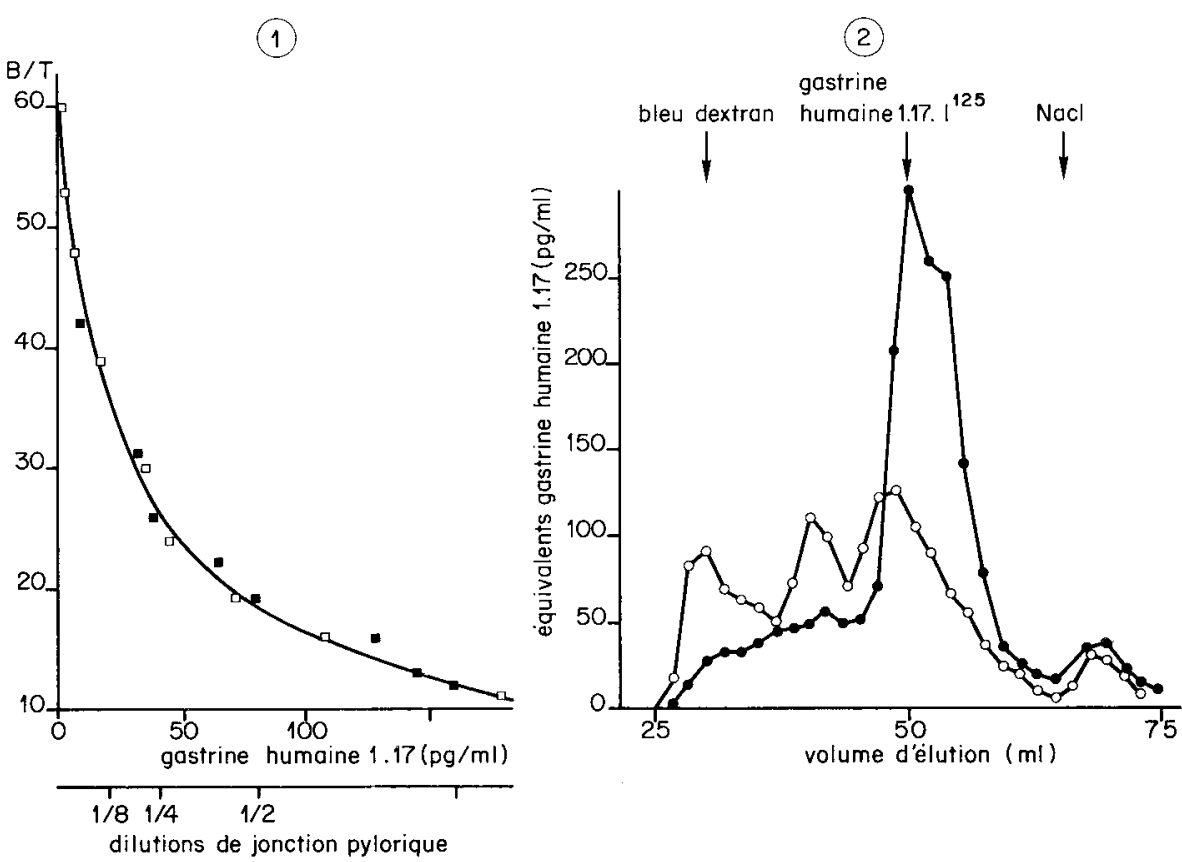

FIG. 1. - Etude de la dilution d'un extrait de jonction pylorique de poulet (Blair et al., 1961) dans un dosage radioimmunologique de gastrine utilisant un anticorps antigastrine humaine 1.17 et de la gastrine humaine 1.17 comme standard et comme traceur. Le rapport de la $1^{125}$ gastrine humaine 1.17 liée (B) à la gastrine totale $(T)$ est mesuré en fonction de concentrations croissantes de gastrine humaine 1.17 froide (de 0 à $200 \mathrm{pg} / \mathrm{ml}$ ) ou d'extrait de jonction pylorique de poulet (de 40 à $700 \mu \mathrm{g} / \mathrm{ml}$ ). $\square$ : gastrine humaine $1.17 ; \boldsymbol{\square}$ : jonction pylorique aviaire.

FIG. 2. - Chromatographie sur sephadex G50 superfine d'un extrait de jonction pylorique de poulet (Blair et ai., 1961) incubé (O) ou non 10 ) en présence de trypsine $10,25 \mathrm{mg} / \mathrm{ml}$ d'extrait pendant $10 \mathrm{~min}$ à $37^{\circ} \mathrm{C}$ puis soumis $10 \mathrm{~min}$ au bain marie bouillant) Dépôt de $3500 \mu \mathrm{g}$ équivalents gastrine humaine 1.17. La calibration de la colonne est effectuée avec du bleu dextran; de la $\mathrm{I}^{125}$ gastrine humaine 1.17 et du $\mathrm{NaCl}$. Flux d'élution: $15 \mathrm{ml} / \mathrm{h}$ à $20{ }^{\circ} \mathrm{C}$, collecte de fractions de $2 \mathrm{ml}$.

2. Essai biologique. - La pentagastrine injectée par voie intraveineuse à des doses allant de 50 à $200 \mu \mathrm{g} / \mathrm{kg}$ augmente la sécrétion gastrique d'acide chlorhydrique des poulets anesthésiés (fig. 3a), son effet est maximum dans les $30 \mathrm{~min}$ qui suivent l'injection et le retour à l'état basal de la sécrétion est observé une 
heure après le début de la stimulation ; une réponse analogue est obtenue après l'injection de 3 doses d'extrait pylorique allant de 6,25 à $25 \mathrm{mg} / \mathrm{kg}$ (fig. 3b). On peut estimer que l'extrait sec étudié dont la teneur en gastrine immunoréactive correspond à $240 \mathrm{pg} / \mathrm{mg}$, se comporte comme s'il contenait $10 \mu \mathrm{g}$ de pentagastrine par $\mathrm{mg}$. La figure $3 \mathrm{~b}$ montre que des poulets privés de jonction pylorique par électrocoagulation (réalisée dans l'heure qui précède l'essai) ont une sécrétion basale fortement réduite mais répondent encore à l'injection de l'extrait pylorique.
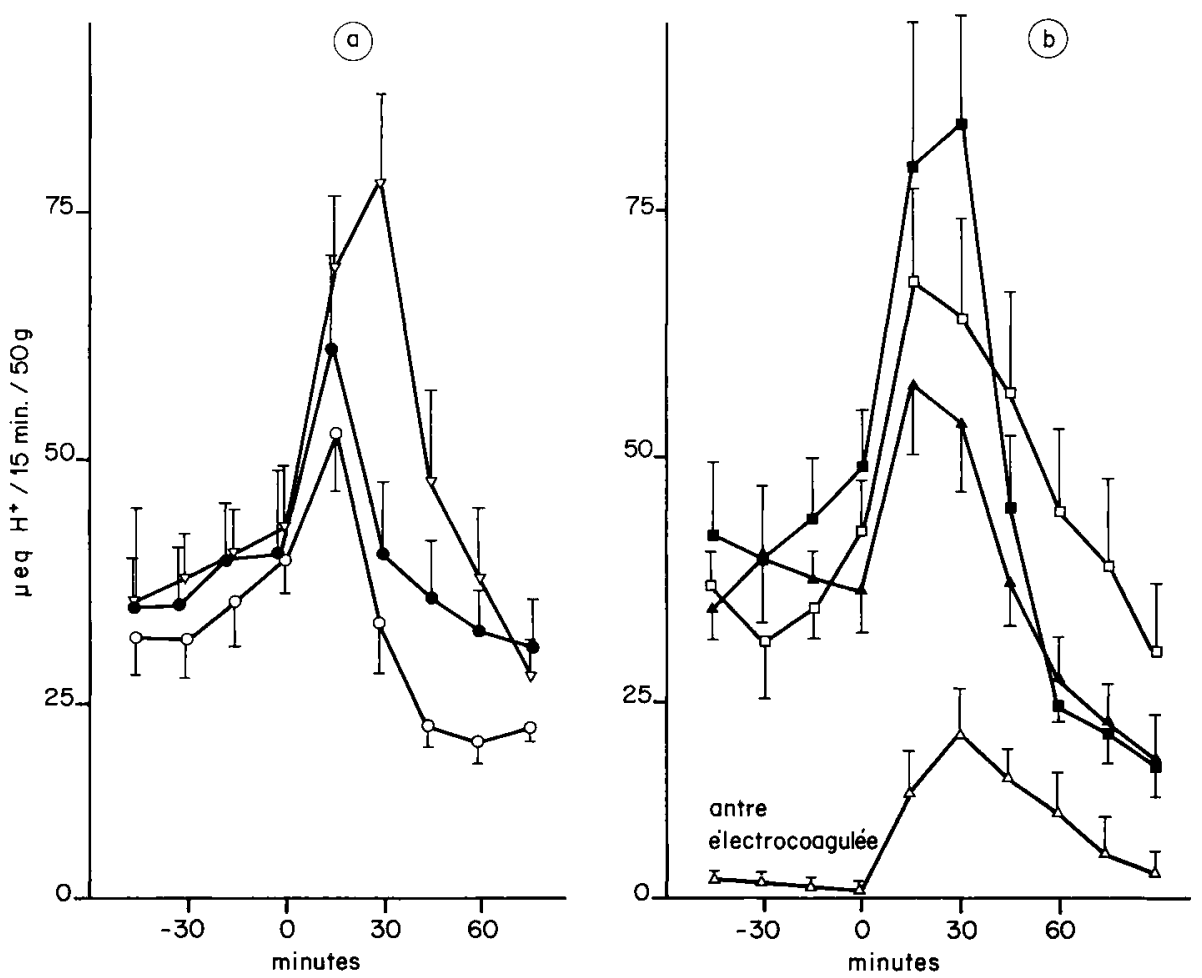

FIG. 3. - Sécrétion gastrique d'acide chlorhydrique induite, chez de jeunes poulets anesthésiés, par injection intraveineuse :

a) de pentagastrine : (O):50 $\mathrm{gg} / \mathrm{kg}, 100 \mu \mathrm{g} / \mathrm{kg},(\nabla): 200 \mu \mathrm{g} / \mathrm{kg}$,

b) d'un extrait de jonction pylorique de poulet (Blair et al., 1961): (\):6,25 $\mathrm{mg} / \mathrm{kg},(\square)$ :

$15 \mathrm{mg} / \mathrm{kg}$, (四): $25 \mathrm{mg} / \mathrm{kg}$, $25 \mathrm{mg} / \mathrm{kg}$.

$-(\Delta)$ : poulets dont la jonction pylorique a été détruite par électrocoagulation, extrait à

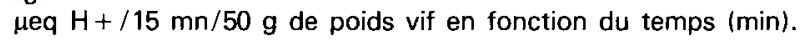

$m+\frac{s}{V n}(n=8)$.

\section{Discussion.}

On ne connaît ni la structure ni l'activité biologique de la gastrine aviaire et jusqu'à présent seules deux équipes utilisant un dosage radioimmunologique 
hétérologue ont pu apporter quelques informations. Larsson et al. $(1974,1977)$ en utilisant différents antisérums antigastrine humaine 1.17 suggèrent qu'il existe de fortes différences entre la gastrine aviaire et la gastrine des mammifères. En effet sur plusieurs antisérums essayés, un seul présente une réaction croisée avec des extraits pyloriques de poulet. Cet antisérum qui reconnaît la partie terminale de la gastrine humaine 1.17 ne donne cependant pas de résultats cohérents pour des dilutions différentes d'extraits, ni ne détecte de forme moléculaire de poids supérieur à celui de la gastrine 1.17 ; les autres antisérums, spécifiques des régions $\mathrm{NH}_{2}$ terminales et moyennes de la gastrine humaine 1.17 ou de la molécule dans son ensemble ne révèlent aucune activité immunologique. Dockray (1979) confirme par ailleurs que les facteurs gastriniques présents dans la muqueuse antrale de dinde possèdent une région terminale analogue mais non identique à celle de la gastrine humaine 1.17 et de la cholecystokinine (CCK8).

L'anticorps que nous avons utilisé n'est pas dirigé exclusivement contre la partie $C$ terminale de la gastrine (puisque la gastrine à 34 acides aminés et la pentagastrine sont moins reconnues : voir matériel et méthodes) et contrairement aux anticorps utilisés par Larsson (1974), il reconnaît dans la muqueuse pylorique de poulet une substance dont le comportement immunologique (test de parallélisme) est semblable à celui de la gastrine humaine 1.17 et dont la mesure quantitative est possible. Les caractéristiques de l'antisérum employé permettent d'envisager pour la gastrine de poulet, l'existence de déterminants antigéniques communs avec la gastrine humaine 1.17 autres que les 5 acides aminés C terminaux. La spécificité du dosage est par ailleurs confirmée par la localisation prépondérante de gastrine immunoréactive dans la zone pylorique (tabl. 1). Les activités mesurées sont plus faibles que celles obtenues chez les mammifères sur des extraits antraux préparés dans des conditions analogues aux nôtres [Malmstrom et Stadil, 1975, donnent les valeurs suivantes : (en équivalents gastrine humaine 1.17 ) chez I'homme : $28 \mu \mathrm{g} / \mathrm{g}$; le chat : $1,7 \mu \mathrm{g} / \mathrm{g}$; le lapin : $0,8 \mu \mathrm{g} / \mathrm{g}$ )] mais comparables aux activités mesurées chez les amphibiens (Gibson et al., 1976 : de 0,002 à $0,04 \mu \mathrm{g}$ équivalent gastrine humaine $1.17 \mathrm{par} \mathrm{g}$ d'extrait antral). Cet anticorps a en outre mis en évidence trois formes moléculaires qui après digestion trypsique sont transformées comme chez les mammifères (Rehfeld, 1972) en une seule forme de poids moléculaire comparable à celui de la gastrine humaine 1.17 (PM 2150); ce résultat permet de supposer que la gastrine est synthétisée sous forme de précurseurs de poids moléculaire élevé chez le poulet comme chez les mammifères ; il est cependant impossible de dire quelles sont les proportions relatives des différentes formes puisque l'antisérum ne les reconnaît vraisemblablement pas toutes également chez le poulet comme chez I'homme.

Nous avons pu montrer que les extraits pyloriques possèdent une activité biologique gastrine-like chez le poulet ; l'effet sécrétoire sur les cellules pariétales est bien direct puisqu'il se manifeste encore chez des poulets privés de jonction pylorique. Cependant le manque de correspondance entre les concentrations " immunologiques » et les activités biologiques laisse supposer d'une part que la gastrine aviaire est plus active que la pentagastrine chez le poulet comme chez les mammifères (Walsh 1981) (son action pouvant en outre avoir été potentialisée 
par d'autres substances sécrétagogues présentes dans les extraits pyloriques) et/ou d'autre part que le système radioimmunologique utilisé reconnaît incomplètement la gastrine de poulet (en rappelant toutefois qu'il y a parallélisme entre les dilutions d'extrait pylorique et celles d'étalon gastrine humaine 1.17).

Reçu en octobre 1982. Accepté en mai 1983.

\section{Références}

ANDREW A., 1976. Endocrine cells of the stomach of chicks around the time of hatching. Cell Tiss. Res., 172, 553-561.

BLAIR E. L., HARPER A. A., LAKE H. J., REED W. D., SCKATCHERD T., 1961. A simple method of preparing gastrin. J. Physiol. (Lond.), 156, 11-12.

BLAIR E. L., SHERRAT H. J. A., WOOD D. D., 1967. The subcellular distribution of gastrin and secretin in the mucosa of the small intestine. Biochem. J., 104, $54 \mathrm{P}$.

BURHOL P. G., 1971. Gastric secretion in the chicken. Scand. J. Gastroenterol., 6, supp. 11. 41-55.

DOCKRAY G. J., 1975. Comparative studies on secretin. Gen. comp. Endocrinol., 25, 203-210.

DOCKRAY G. J., 1979. Cholecystokinin-like peptides in avian brain and gut. Experientia, 35/5, 628-630.

GABE M., 1972. Données histologiques sur les cellules à gastrine des sauropsidés. Archives Anat. microscop., 61, 175-200.

GIBSON R. G., MIMAS A. A., COLVIN M. W., HIRSCHOWITZ B. I., 1976. The search for submammalian gastrins : the identification of amphibian gastrin. Proc. Soc. exp. Biol. Med., 53, 284-288.

GOTO Y., WATANABE F., 1976. Gastric acid secretory responses of acute gastric fistula preparation in anesthetized young chickens. Experientia, 32/7, 946-948.

HAZELWOOD R. L., 1980. The avian gastro-enteric-pancreatic system : structure and function, 231-250. A. EPPLE, M. H. STETSON. In Avian endocrinology. Acad. Press Inc.

KETTERER M., RUOFF M. J., SEWING K. F., 1973. Do chickens have gastrin like compounds? Experientia, 29, 2096.

KOKAS E., PHILLIPS G. L., BRUNSOU W. D., 1967. The secretory activity of the duodenum in chickens. Comp. Biochem. Physiol., 22, 81-90.

KOKAS E., KAUFMAN S. H., LONG J. C., 1971. Effect of glucagon on gastric and duodenal secretion in chicken. Z. Vergl. Physiologie, 74, 315-325.

LARSSON L. I., SUNDLER F., HAKANSON R., RHEFELD J. F., STADIL F., 1974. Distribution and properties of gastrin cells in the gastrointestinal tract of chicken. Cell Tiss. Res., 154, 409-421.

LARSSON L. I., REHFELD J. F., 1977. Characterization of antral gastrin cells with region specific antisera. J. Histochem. Cytochem, 25, 1317-1321.

LINARI G., BALDIERI-LINARI M., 1976. On the mechanism of action of bombesin on gastric and pancreatic secretion of the chicken. Arch. int. Pharmacodyn., 224, 283-290.

MALMSTRÖM J., STADIL F., 1975. Measurement of immunoreactive gastrin in gastric mucosa. Scand. J. Gastroenterol., 10, 433-439.

OLOWO-OKORUN M. O., AMURE B. O., 1973. Gastrin activity in the chicken proventriculus. Nature, 246, 424-425.

POLAK J. M., PEARSE A. G. E., ADAM S. C., GARAUD J. C., 1974. Immunohistochemical and ultra structural studies on the endocrine polypeptide (APUD) cells of the avian gastrointestinal tract. Experientia, 30, 564-567.

REHFELD J. F., 1972. The components of gastrin in human serum. Gel filtration studies on the molecular size of immunoreactive gastrin. Biochim. biophys. Acta, 285, 364-372. 
RUOFF H. J., SEWING K. P., 1970. Histidindecarboxylase und gastrin in oberen Verdanungstrakt des Hühns. Naunyn. Schmiedebesgs. Arch. Pharmakol., 265, 301-309.

SEWING K. F., RUOFF M. J., 1972. Control of gastric acid secretion in chickens. Acta hepato gastroenterol., 19, 296-300.

WALSH J. H., 1981. Gastrointestinal hormones and peptides, 59-144. L. R. JOHNSON, Physiology of the gastrointestinal tract. Raven Press, N. Y.

WALSH J. H., ISENBERG J. I., ARNFIELD J., MAXWELL V., 1976. Clearance and acid stimulating action of human big and little gastrin in duodenal ulcer subjects. J. clin. Invest., 57, 1125-1131. 